\title{
Few-layer borophene prepared by mechanical resonance and its application in terahertz shielding
}

Zhixun Zhang, † Ming Zhou, *t, Taohong Zhang,\& Mingyang Yangt, Qingfeng Yangt, Jianwen Yut, Yibo Zhangt

†State Key Laboratory of Tribology, School of Mechanical Engineering, Tsinghua University, Beijing 100084, China.

łKey Laboratory for Advanced Materials Processing Technology, Ministry of Education, P.R. Beijing 100084, China.

§Department of Computer, School of Computer and Communication Engineering University of Science and Technology Beijing (USTB), Beijing 100083, China.

E-mail: zhouming@tsinghua.edu.cn 

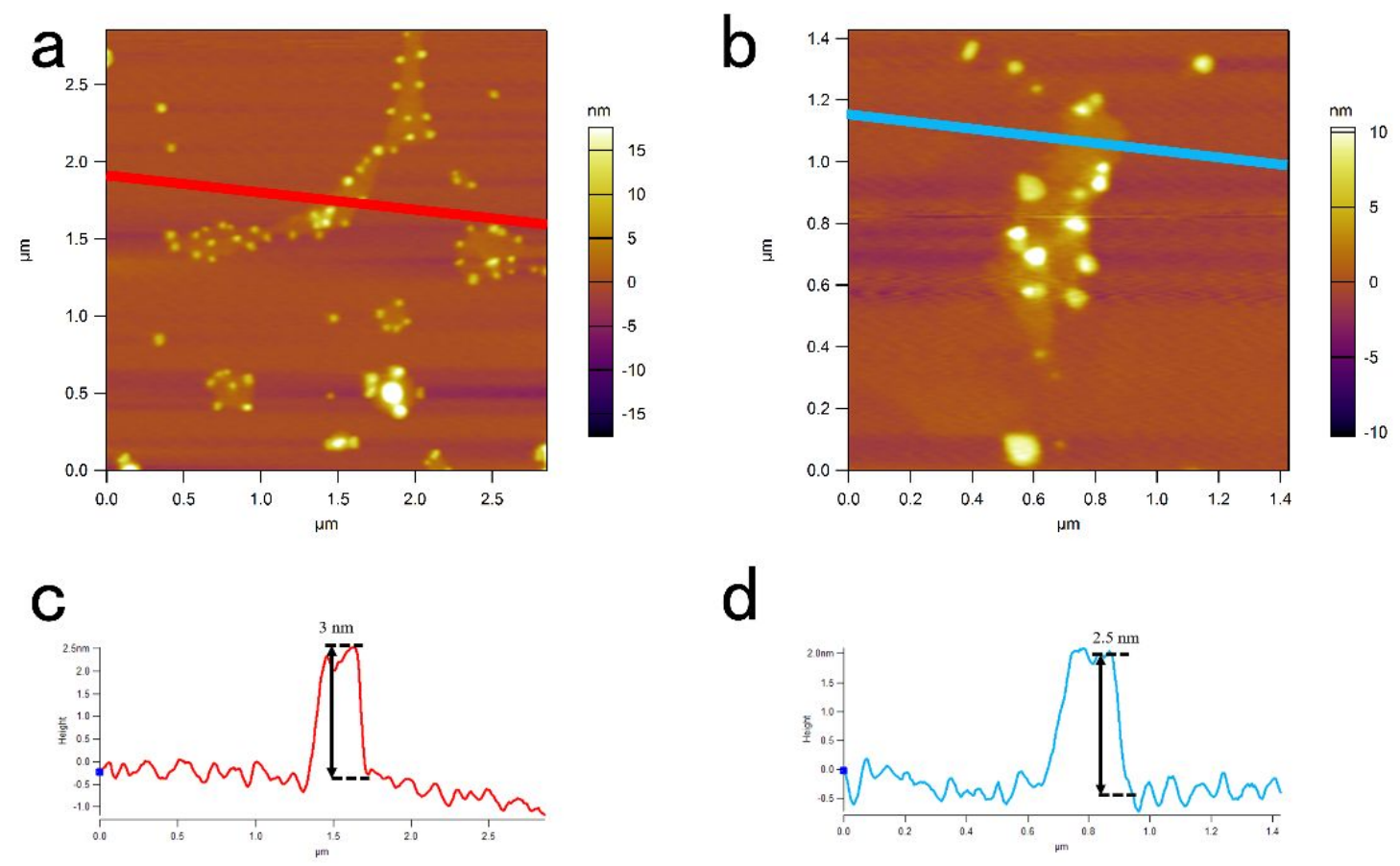

Figure S1. The same experimental results repeated by the optimal experiment conditions under AFM. The thickness of few-layer borophene is less than $4 \mathrm{~nm}$. (a, b) 2D images of few-layer borophene on silver nanowire crystal planes. (c) Height change of the red line in (a). (d) Height change of the red line in (b).
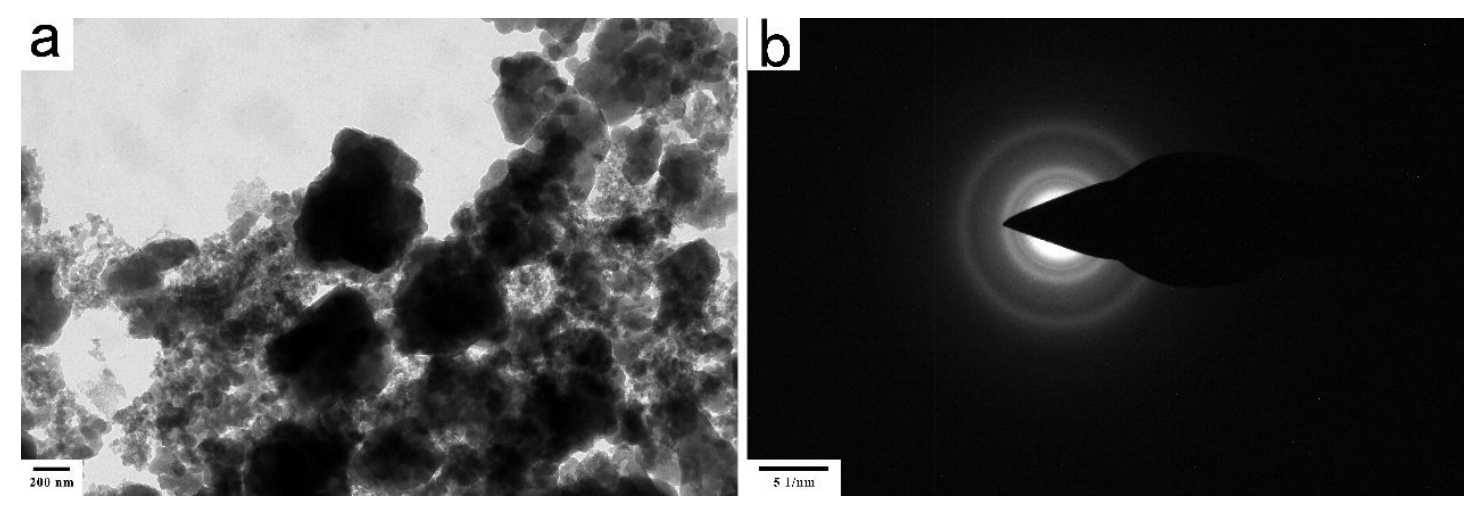

Figure S2. Amorphous structure of amorphous element boron. (a) TEM image of amorphous element boron. (b) Selected area electron diffraction image of 
amorphous element boron indicates the raw material is amorphous structure.

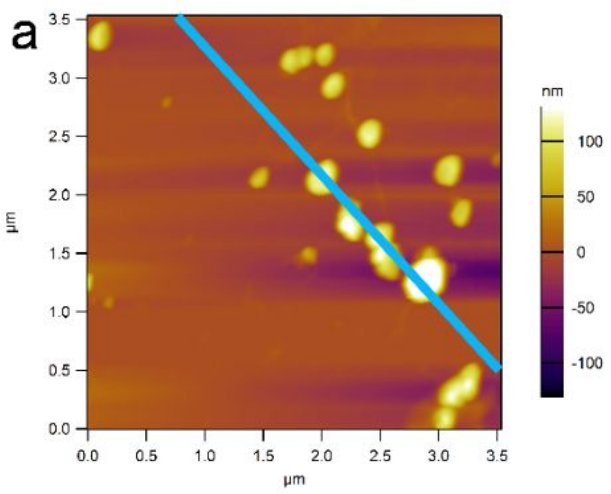

b
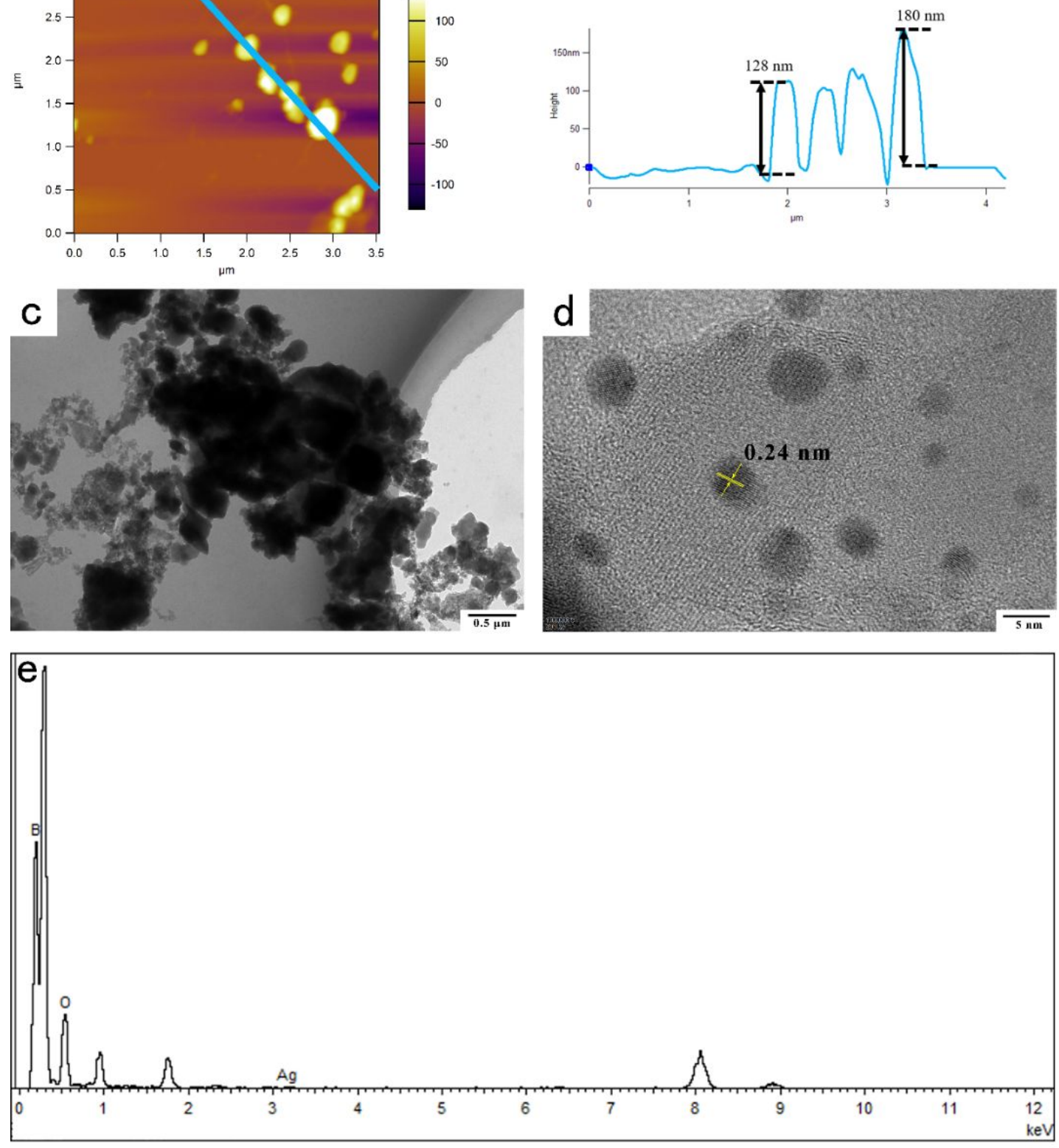

Figure S3. Products prepared without silver nanowires and characterization of silver nanoparticles in the few-layer borophene. (a) AFM image of the product prepared without silver nanowires. (b) Height change of the red line in (a). (c) 
TEM image of the product prepared without silver nanowires. (d) The crystal structure of silver nanoparticles in the few-layer borophene characterized by HRTEM. The lattice constant of the silver nanoparticles is $0.24 \mathrm{~nm}$. (e) Energy spectrum of the few-layer borophene with silver nanoparticles in the area. 


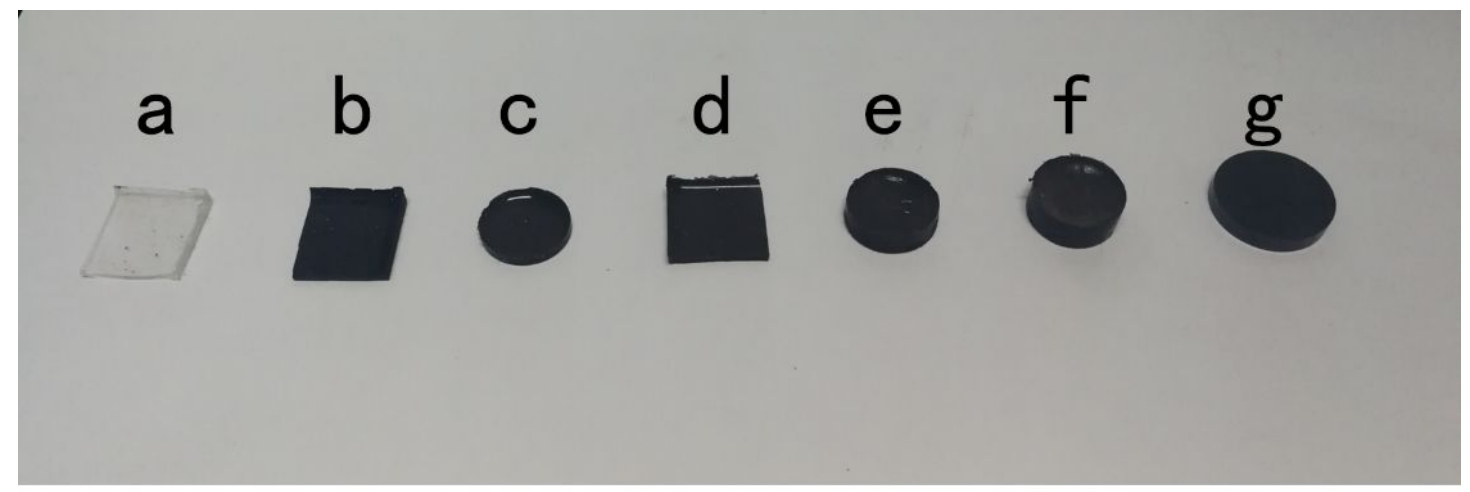

\section{h Parabolic reflector}

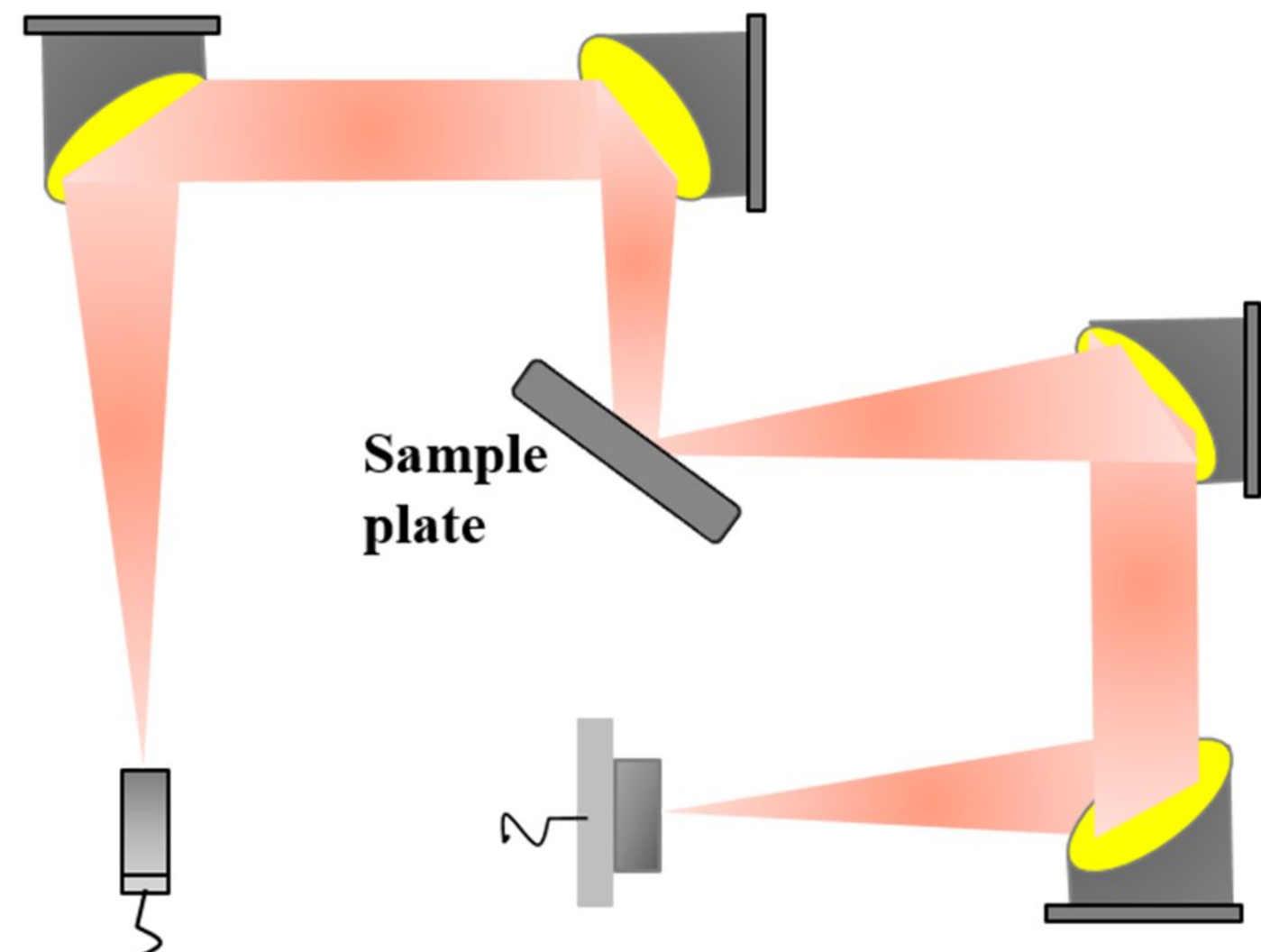

\section{Transmitter Detector}

Figure S4. Photo image of terahertz shielding and stealth samples and the schematic diagram of reflected wave detection system. (a) Blank PDMS. (b) Boron doping PDMS. (c-f) Borophene doping PDMS with different thickness $(0.5 \mathrm{~mm}, 1 \mathrm{~mm}, 2.5 \mathrm{~mm}$ and $5 \mathrm{~mm}) .(\mathrm{g})$ 
The tableting sample of borophene powders. (h) The schematic diagram of reflected wave detection system.

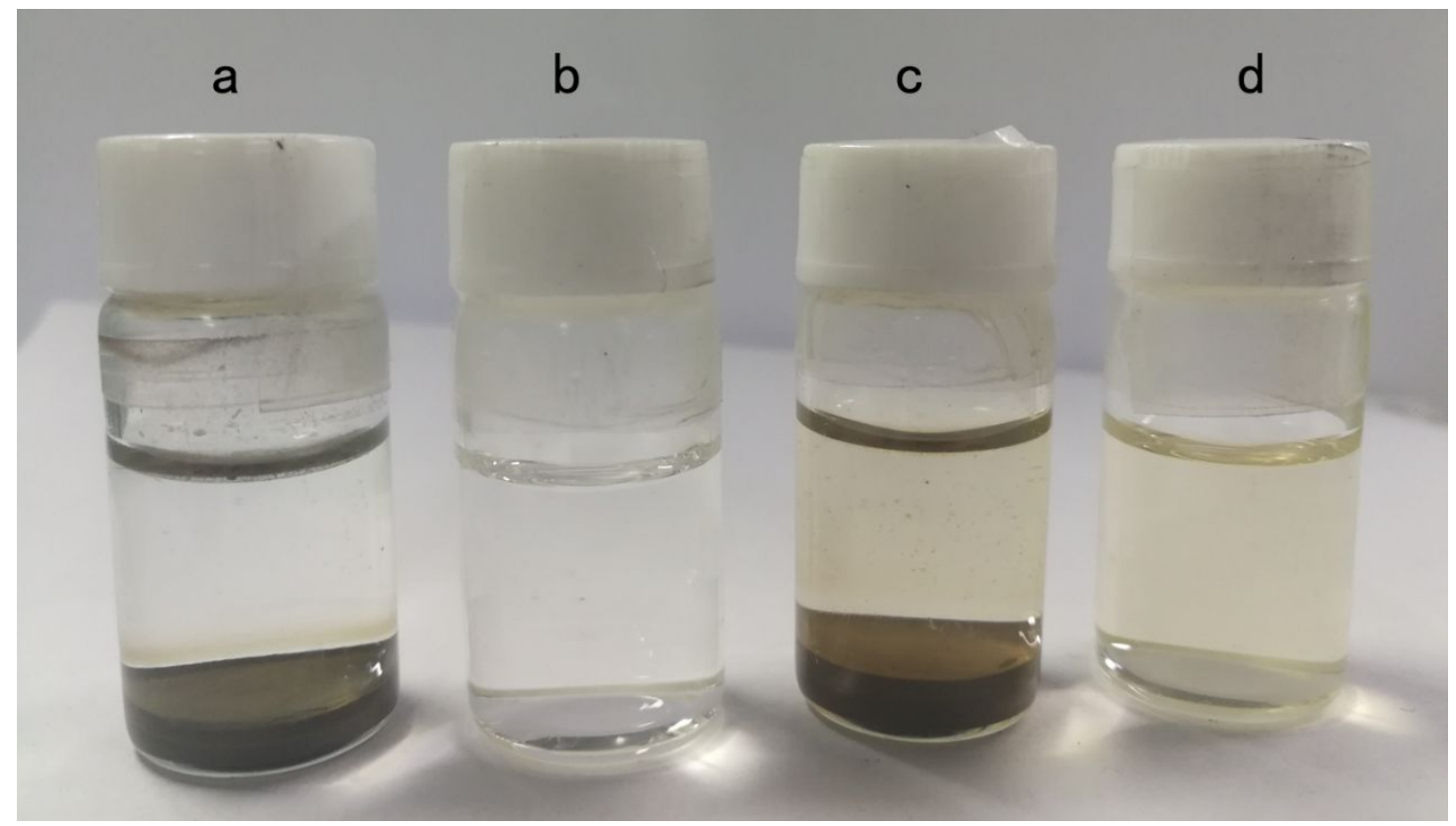

Figure S5. Photo image of amorphous element boron and borophene dispersion in water. (a)

Amorphous element boron dispersion in water after six months' rest. (b) The supernatant from (a). (c) Borophene dispersion in water after six months' rest. (d) The supernatant from (c).

We found that there was nothing in the supernatant because amorphous element boron was all precipitation in water after six months' rest. And the supernatant of borophene appeared brown and yellow because of its fine dispersion in water.

Table S1. Element content of prepared samples. 


\begin{tabular}{ccc}
\hline Element & Weight percentage & Atomic percent \\
\hline B K & 83.00 & 94.82 \\
O K & 3.25 & 2.51 \\
Cu K & & \\
\hline Total quantity & 13.75 & 2.67 \\
& & \\
\hline
\end{tabular}

Table S2. Element content of prepared samples.

\begin{tabular}{ccc}
\hline Element & Weight percentage & Atomic percent \\
\hline B K & 90.60 & 93.60 \\
O K & 9.13 & 6.37 \\
Ag K & & 0.03 \\
Total quantity & 0.27 & \\
\hline
\end{tabular}

a These element contents of boron from energy spectrum could give qualitative description of element composition and content.

Table S3. Formation energies for free-standing and substrate-supported borophene. 


\begin{tabular}{ccc}
\hline & Large substrate & Smaller substrate \\
& {$[$ [eV/atom] } & [eV/atom $]$ \\
\hline$E_{\mathrm{FB}}$ & 6.29 & 6.31 \\
$\mathrm{E}_{\mathrm{EB}}$ & & 6.49 \\
& 6.71 & \\
$\mathrm{E}_{\mathrm{A}}$ & 0.42 & 0.18 \\
\hline
\end{tabular}

a $\mathrm{E}_{\mathrm{FB}}$ is the formation energy per atom for free-standing $\alpha$-sheet borophene; $\mathrm{E}_{\mathrm{EB}}$ is the formation energy per $\mathrm{B}$ atom for substrate-supported borophene. $\mathrm{E}_{\mathrm{A}}$ is adhesion energy of boron sheet per B atom. 